\title{
Journal of Ophthalmic Inflammation and Infection: why a new journal on ocular inflammation?
}

\author{
Narsing A. Rao
}

Received: 29 September 2010 / Accepted: 1 October 2010 /Published online: 10 November 2010

(C) The Author(s) 2010. This article is published with open access at Springerlink.com

The widespread use of the Internet and electronic publishing has contributed to the evolution of open-access journals, including those in the field of ophthalmic research. Such peer-reviewed electronic publications are highly desirable since they encourage the rapid publication of basic and clinical research, unlike the traditional paper journals. Timely publication of research without substantial delay will help ensure the rapid translation of basic and clinical studies to the bedside, introducing diagnostic and therapeutic interventions in the management of complex ocular inflammations and infections. Clearly, online openaccess journals represent the future direction of publishing for the dissemination of biomedical research and the everchanging approaches to diagnosis and therapeutic intervention. Moreover, open-access electronic publications are easily retrieved, without the imposition of financial constraints at either the institutional or the individual level, in both the developed and the developing world. Since infectious diseases are more prevalent in developing countries, access to peer-reviewed electronic journals offers the opportunity for prompt publication of emerging infectious diseases from various geographic regions, as well as for continued education for clinicians practicing in such countries and in the rest of the world. The ability of electronic journals to offer cost-effective, timely publication with abundant high-quality illustrations will enhance patient care by allowing the timely recognition of ophthalmic inflammatory conditions. Emerging imaging technology is transforming the diagnosis and classification of various ophthalmic disorders, including intraocular inflam-

N. A. Rao $(\bowtie)$

Doheny Eye Institute/University of Southern California,

Los Angeles, CA, USA

e-mail: nrao@doheny.org mations, and is providing in vivo illustrations of the pathologic process; the further characterization of ocular inflammation with the aid of imaging methods should advance the current management of inflammatory processes. For such advancement, electronic journals offer the publication of images with minimal or no cost to authors.

The above advantages of electronic publication will no doubt have a significant impact in the ophthalmic field in general and in ocular inflammation and infection in particular. This impact is becoming obvious since openaccess articles are cited more often than non-open-access traditional journals. The increased use translates into a substantial increase in the impact of a publication.

Under the leadership of Professors Phuc LeHoang, Jorge Alio, and Talin Barisani-Asenbauer, the International Ocular Inflammation and Infection society (IOIS) decided to initiate an electronic journal to the benefit of members and non-members interested in the field of ophthalmic inflammation and infection. An election was held in 2007, during the annual IOIS business meeting held during the IOIS congress in Paris, France, to choose the editor-in-chief for such a journal

As editor-in-chief of the journal, my first task was to select the editorial board members. This was not an easy task since there were several IOIS members and others with remarkable expertise. Thus, the selection of the current board members reflects their expertise, the fairness of their reviewing process, and their high ethical standards. Moreover, to reflect the global nature of the new journal, we have attempted a fair distribution of the editorial board to represent different geographical areas. All of the board members enthusiastically support the common goal of improving patient care in ophthalmic inflammation and infections through the prompt review of articles that are clinically relevant and preferably evidence-based. 
Our mission in launching this new journal is to provide fair review and prompt publication of relevant articles, including high-quality images, without cost to authors. Moreover, priority will be given to expedited publication of those articles which provide cutting-edge clinical details for improving the care of patients with ocular inflammation and infections. To accomplish these goals, the submitted manuscripts will be reviewed briefly by the editor-in-chief for relevance of content and then assigned to expert reviewers from among a pool of IOIS and other ocular inflammation society members. If required, additional experts outside the field of ocular infection will be invited as reviewers. Every attempt will be made to obtain a minimum of two reviews, and based on the reviewers' recommendations, the manuscript will be graded as $\mathrm{A}$ - accept as is; $\mathrm{B}$ - minor revision required; $\mathrm{C}$-major revision required; or D-reject. The editor-in-chief will go over the reviews for fairness and make a final judgment. The entire process from submission to final judgment should be around 6-8 weeks, and the accepted papers will appear in the electronic journal within 3 months. Although we will strive to deliver the journal once a month, during the next 12 24 months, we will limit the number of issues to four until the current pace of high-quality manuscripts submission is enhanced.

Narsing A. Rao, MD

Editor-in-Chief

Journal of Ophthalmic Inflammation and Infection

Open Access This article is distributed under the terms of the Creative Commons Attribution Noncommercial License which permits any noncommercial use, distribution, and reproduction in any medium, provided the original author(s) and source are credited. 